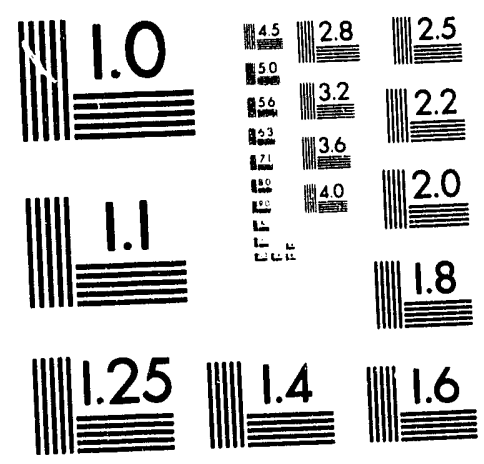



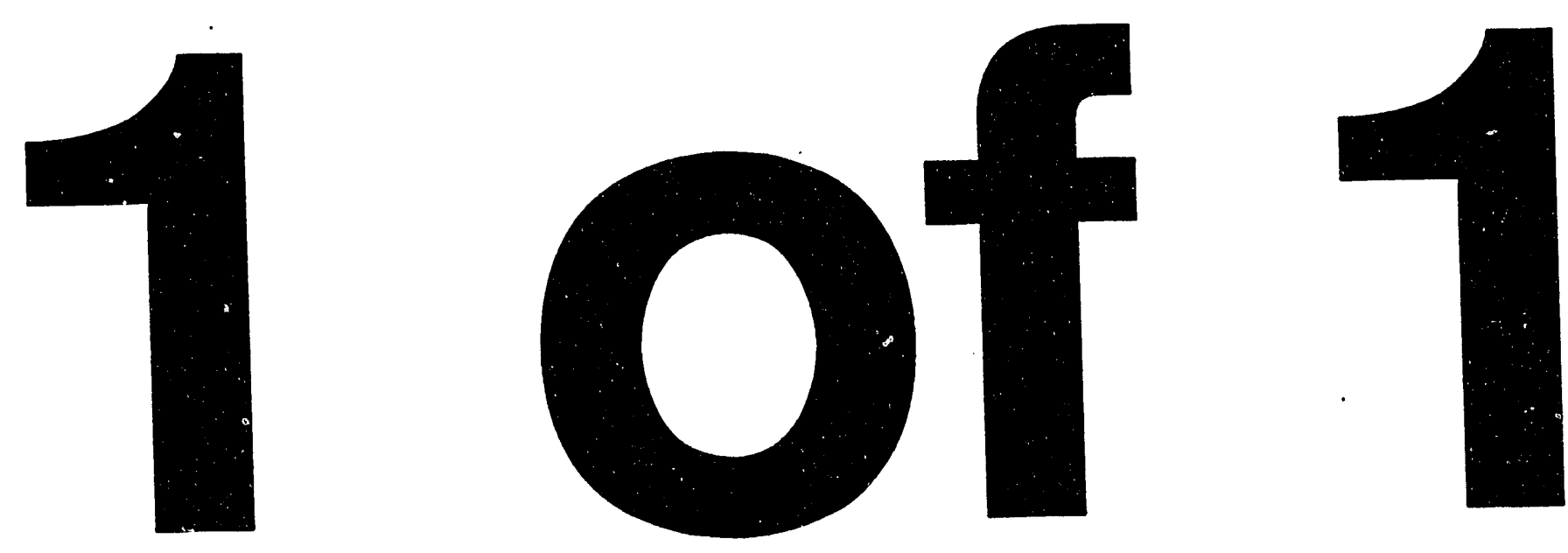


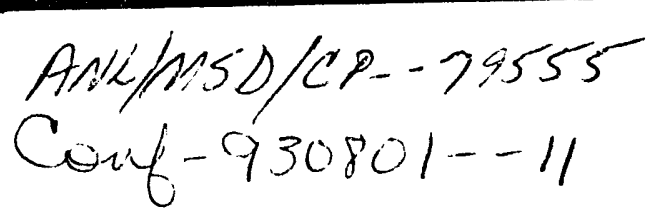

\title{
DIMENSIONAL CROSSOVER IN THE TORQUE IN A LAYERED
}

SUPERCONDUCTOR*

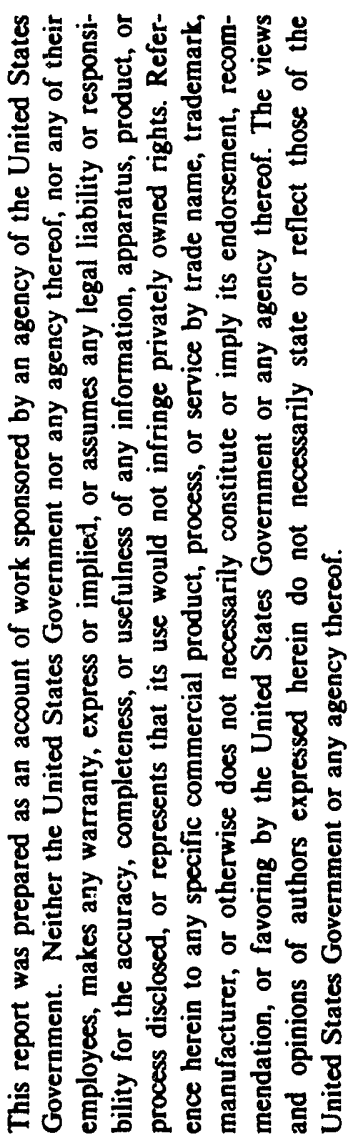

\author{
R. A. Klemm \\ Materials Science Division \\ Argonne National Laboratory \\ Argonne, IL 60439
}

\begin{abstract}
The submitted manuscript has been authored by a contractor of the U. S. Government under contract No. W-31-109-ENG-38.

Accordingly, the U.S. Government retains a nonexclusive, royalty-free license to publish or reproduce the published form of this contribution, or allow others to do so, for
\end{abstract} U.S. Government purposes.

APRIL 1993

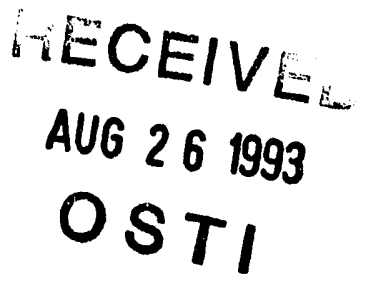

/rlp

Submitted to International Conference LT XX on Low Temperature Physics, Eugene, OR, August 4-11, 1993.

* Work Supported by the U.S. Department of Energy, BES-Materials Sciences, under Contract \#W-31-109-ENG-38. 


\section{Dimensional crossover in the torque in a layered superconductor}

\section{R. A. Klemm}

Materials Science Division, Argonne National Laboratory, Argonne, IL 60439

The procedure of Bulaevskir, Ledvij, and Kogan for evaluating the line energy of a single, straight vortex in the Lawrence-Doniach model in the linearized phase-only approximation is modified to take accurate account of the vortex core cross-sections. Dimensional crossover effects are found to be pronounced, with oscillations in the angular dependence of the torque for $\theta \simeq \pi / 2$ and $T \approx T^{*}<T_{c}$.

\section{INTRODUCTION}

The Lawrence-Doniach (LD) model has been used extensively in treating the superconducting properties of layered superconductors [1,2]. Recently, Bulaevskiî, Ledvij, and Kogan (BLK) [3] showed that the line energy of a single vortex in the LD model could be calculated by assuming the amplitude of the order parameter was a constant outside of the vortex core, and by neglecting non-linearities in the order parameter phase $\phi$. BLK assumed the vortex to form a straight line at an angle $\theta$ with respect to the $c$-axis, with a periodic set of topological defects in $h$ at the intersections of the vortex line with the superconducting layers. In the absence of Josephson coupling between the layers, the vortices are pancakes [4], with circular core crosssections at the superconducting layers, as assumed by BLK. Such a situation is applicable in the $2 \mathrm{D}$ regime $T<<T^{*}<$ $T_{c}$, where $T^{*}$ is the dimensional crossover temperature given by $\xi_{\perp}\left(T^{*}\right)=s / \sqrt{2}[2]$. However, it is not correct in the (3D) London regime $T^{*}<T<T_{c}$, and cannot therefore properly address the question of dimensional crossover effects in the vortex line energy $\epsilon(\theta, T)$ and in the magnetic torque $\tau(\theta, T)=\partial \epsilon / \partial \theta$. In general, the vortex core cross-section is given by the lines of constant order parameter amplitude [5], which vanishes at the core centers. To investigate the role of dimen- sional crossover on $\tau(\theta, T)$, we have therefore modified the BLK core cutoff to account for finite Josephson coupling. As shown below, such a treatment reduces to the correct London limit $[5,6]$ for $T^{*}<$ $T \simeq T_{c}$, and to the $2 \mathrm{D}$ limit of BLK for $T<<T_{*}$. In addition, it give striking oscillations in $\tau(\theta)$ for fixed $T \approx T^{*}$, which may serve as a fingerprint for the intrinsic superconducting parameters of the material under study.

\section{VORTEX LINE ENERGY}

Using the linearized phase-only LD model, BLK found the line energy of a single vortex to be given by $[3 ; 5]$

$$
\epsilon=\frac{\Phi_{0}^{2}|\cos \theta|}{32 \pi^{3}} \int d^{2} \mathbf{k} \frac{k_{x}^{2} G_{\perp}+Q^{2} G_{\|}}{k_{x}^{2} G_{3} G_{\|}},
$$

where $G_{\|, \perp}\left(k_{x}, k_{y}\right)=1+\lambda_{\|, \perp}^{2}\left(k^{2}+Q^{2}\right)$, $G_{3}\left(k_{x}, k_{y}\right)=1+\lambda_{\|}^{2} Q^{2}+\lambda_{\perp}^{2} k^{2}, Q^{2}=$ $\left(2 / s^{2}\right)\left[1-\cos \left(k_{x} s \tan \theta\right)\right], \lambda_{\|}$is the penetration depth for $\mathbf{H} \| \hat{\mathbf{c}}, s$ is the $c$-axis repeat distance, $\lambda_{\perp}=\gamma \lambda_{\|}, \gamma=(M / m)^{1 / 2}$ $\geq 1$ is the anisotropy factor, $\Phi_{0}$ is the flux quantum, and $k^{2}=k_{x}^{2}+k_{y}^{2}$. The integration over $\mathbf{k}$ is restricted to the regions outside the cores. As shown elsewhere [5], this restriction can be accurately obtained from the linearized order parameter amplitude equation below $T_{c}$, 
using transformation procedures $[5,6]$,

$$
k^{2}+Q^{2} / \gamma^{2} \leq 2 \xi_{\|}^{-2}
$$

where $\xi_{\|}=\gamma \xi_{\perp}$ is the Ginzburg-Landau coherence length parallel to the layers. It is straightforward to evaluate the integral over $k_{y}$ analytically. In evaluating the integral over $k_{x}$, we let $k_{x}=\gamma^{2} z / s \tan \theta$. In the limit $\kappa_{\perp} \equiv \lambda_{\|} / \xi_{\|}>>1$, we find $\epsilon=\left(\bar{\epsilon}_{1}+\bar{\epsilon}_{2}\right) \Phi_{0}^{2} /\left(16 \pi^{2} \lambda_{\|}^{2}\right)$, where

$$
\begin{aligned}
& \bar{\epsilon}_{1}=|\cos \theta| \ln \left(\frac{1+|\cos \theta|}{\gamma[\mathcal{A}(\theta)+|\cos \theta|]}\right) \\
& \bar{\epsilon}_{2}=\int_{1 / \kappa^{\prime}}^{\infty} \frac{2 d z \mathcal{A}(\theta) \Theta(h) \sqrt{f} \tan ^{-1} \sqrt{h / f}}{\pi z^{2}}
\end{aligned}
$$

$\mathcal{A}^{2}(\theta)=\cos ^{2} \theta+\gamma^{-2} \sin ^{2} \theta, 1 / \delta(\theta)=1+$ $\gamma^{2} \cot ^{2} \theta, f(z)=z^{2}[1-\delta(\theta) g(z)], g(z)=$ $1-2 z^{-2}(1-\cos z), \kappa^{\prime}=2 \delta^{1 / 2}(\theta) \lambda_{\|} / s$, $h(z, \theta)=2\left(s / \xi_{\perp}\right)^{2} \delta(\theta)-f(z)$, and $\Theta(x)$ is the Heaviside step function. The main contribution to $\bar{\epsilon}_{2}$ arises from the region $1 / \kappa^{\prime} \leq z \leq z_{0} \equiv \min f^{-1}\left[2\left(s / \xi_{\perp}\right)^{2} \delta(\theta)\right]$. The torque $\tau(\theta)=\partial \epsilon / \partial \theta$ can then be found in closed (integral) form.

We have evaluated the line energy and the torque numerically, keeping only the region $1 / \kappa^{\prime} \leq: \leq \leq z_{0}$ for simplicity. In Fig. 1, we have plotted the (dimensionless) torque $\bar{\tau}(\theta)=\partial\left(\bar{\epsilon}_{1}+\bar{\epsilon}_{2}\right) / \partial \theta$ versus $2 \theta / \pi$ for $\gamma=100, \kappa_{\perp}=1000$, and various values of $r^{\prime}=\sqrt{2} s / \xi_{\perp}(T)$. For $r^{\prime} \leq 2.0, \bar{\tau}(\theta)$ closely approximates the anisotropic London result [5-7]. In these cases, $z_{0} \leq \pi$. Dimensional crossover occurs precisely at $r^{\prime}=2.0$. For $r^{\prime}=$ 2.1 , however, $z_{0}(\theta) \geq \pi$ for $\theta$ sufficiently close to $\pi / 2$, leading to divergences in the torque, arising from step discontinuities in $\bar{\epsilon}(\theta)$. With increasing $r^{\prime}$ (or decreasing $T$ ), fewer divergences are apparent, but remnants of them persist as oscillations. Well below $T^{*}$, the $r^{\prime}=20.0$ curve shows that the oscillations are still present, but very small. Although we expect thermal fuctuations and finite sample mosaics to smear out the extremely narrow divergences, these oscillations should be observable, and could serve as a fingerprint for the particlar material parameters of the layered superconductor under study.

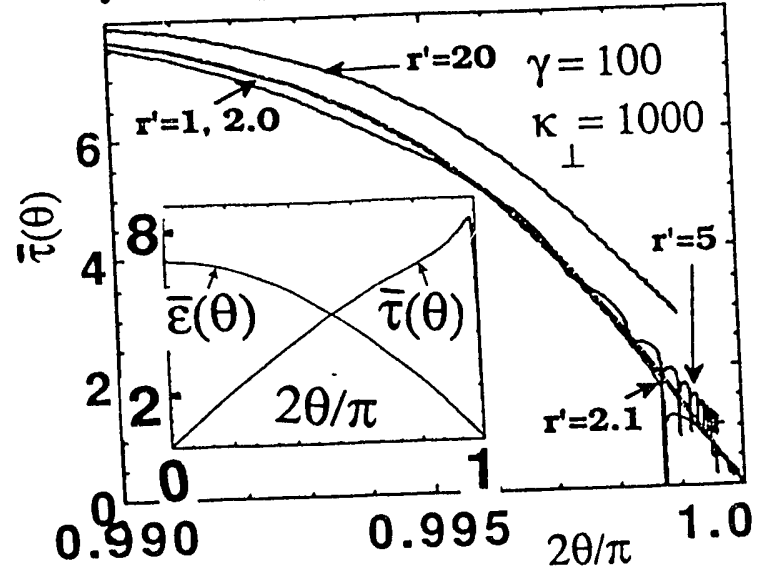

Fig. 1. Plot of $\bar{\tau}(\theta)$ for $\gamma=100, \kappa_{\perp}=$ 1000 , and $r^{\prime}=\sqrt{2} s / \xi_{\perp}$ values $1.0,2.0$, $2.1,5.0$, and 20.0 , for $0.99 \leq 2 \theta / \pi \leq 1$. Inset: Same parameter plots of $\bar{\epsilon}(\theta)$ and $\bar{\tau}(\theta)$ for $0 \leq 2 \theta / \pi \leq 1$.

This work was supp - ited by the USDOE under contract W-31-109-ENG-38.

\section{REFERENCES}

1. W. E. Lawrence and S. Doniach, LT12 Proceedings, E. Kanda, ed., (Academic, Kyoto, Japan), p. 361 (1972).

2. R. A. Klemm, A. Luther, and M. R. Beasley, Phys. Rev. B 12, 877 (1975).

3. L. N. Bulaevskir, M. Ledvij, and V. G. Kogan, Phys. Rev. B 46, 366 (1992).

4. J. R. Clem, Phys. Rev. B 43, 7837 (1991).

5. R. A. Klemm, Layered Superconductors (Oxford, New York) to be published. 6. R. A. Klemm, Phys. Rev. B 47 (1992) (in press); R. A. Klemm and J. R. Clem, Phys. Rev. B 21,1868 (1980).

7. V. G. Kogan, N. Nakagawa, and S. L. Thiemann, Phys. Rev. B 42, 2631 (1990). 

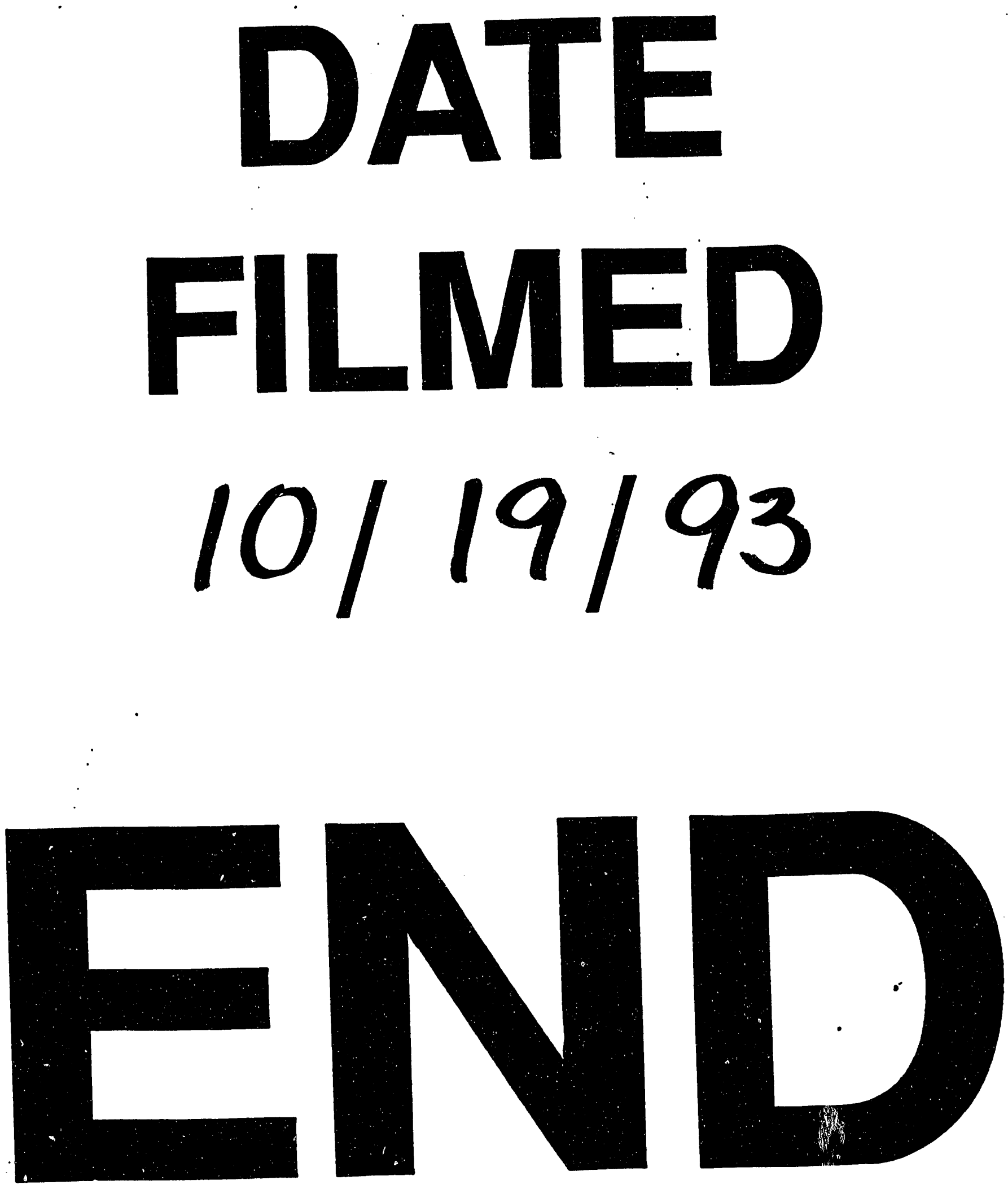
